\title{
Ancrages passifs verticaux et calcul à la rupture
}

\section{P. VEZOLE}

EIFFAGE Construction 3, av. Morane-Saulnier 78140 Vélizy-Villacoublay
La méthode cinématique du calcul à la rupture fournit un éclairage intéressant pour les justifications de stabilité de radiers soumis à des sous-pressions équilibrées par des ancrages verticaux passifs scellés dans le sol sur toute leur longueur. L'article présente les types de mécanismes qu'il convient de prendre généralement en compte pour justifier la longueur des ancrages, notamment lorsque la résistance du sol est caractérisêe par un critère de Coulomb tronqué en traction.

Mots-clés : ancrage, calcul à la rupture, coupure de résistance à la traction.
NDLE: Les discussions sur

cet article sont acceptées jusqu'au 30 septembre 2002.

\section{Passive vertical anchors and Yield design theory}

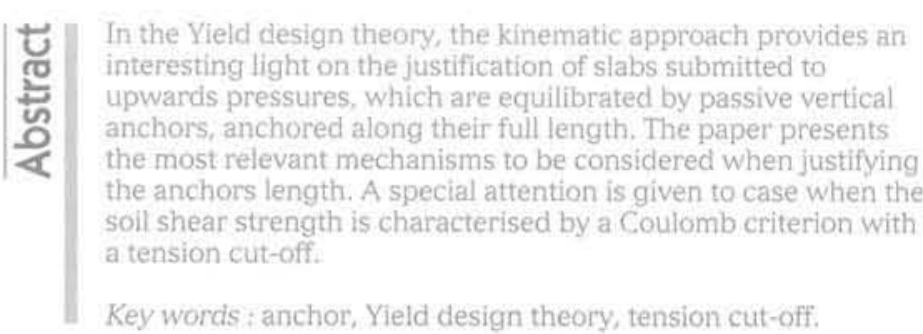




\section{Introduction}

De plus en plus nombreux sont les projets qui comportent des radiers sollicités par des sous-pressions équilibrées au moyen d'ancrages verticaux scellés sur toute leur longueur, que l'on baptise selon le cas tirants, ancrages ou micropieux. Les projeteurs chargés de leur dimensionnement ou de leur justification se réfèrent soit au fascicule 62 titre V du CCTG, soit au DTU 13.2 soit aux Recommandations TA 95, soit encore à l'ENV 1997 (Eurocode 7). On constate que, dans la pratique, les projeteurs se heurtent à des difficultés diverses, d'une part dans l'identification des mécanismes qui conditionnent l'équilibre statique (choix de la longueur des ancrages), et d'autre part pour choisir les coefficients partiels de sécurité et de pondération des justifications de résistance.

La méthode cinématique du calcul à la rupture permet d'apprécier la résistance disponible résultant des forces de pesanteur, du frottement latéral limite et de la résistance au cisaillement du sol.

Le présent article rappelle quelques éléments fondamentaux de la méthode cinématique du calcul à la rupture, puis aborde la physique du problème avant d'évoquer l'application de la méthode des états limites pour la justification des projets.

\section{2}

\section{A propos de la méthode cinématique du calcul à la rupture}

Ce paragraphe s'adresse aux lecteurs qui ne sont pas familiarisés avec la méthode cinématique du calcul à la rupture (méthode malheureusement peu populaire en France) et qui, ignorants des principes de base, ne pourraient qu'éprouver les plus grandes difficultés à comprendre la suite de l'article.

\section{1}

\section{Sol obéissant au critère de Coulomb}

Nous ne prendrons pas le risque de faire un mauvais résumé des ouvrages de référence rédigés par Chen ou par Salencon, dont on ne peut que conseiller la lecture. Nous nous limiterons à quelques indications relatives à des cas de mécanismes de rupture par blocs, en géotechnique, au travers d'exemples simples.

On envisage un mécanisme de géométrie donnée ; un ou plusieurs blocs sont animés par un champ de vitesses virtuelles "cinématiquement admissible » (en 2D, un bloc est soumis à une rotation autour d'un point - une translation étant une rotation autour d'un centre à l'infini - ; en 3D, un champ de vitesses virtuelles affecté à un bloc rigide est une rotation autour d'un axe accompagnée d'une translation parallèle à ce même axe, soit un mouvement de tire-bouchon - une translation ou une rotation en sont des cas particuliers que l'on utilise le plus souvent -); on écrit le bilan des puissances virtuelles : pesanteur, actions diverses, résistances ; le long d'une surface de discontinuité de vitesse flimite entre un bloc en mouvement et le massif immobile ou limite entre deux blocs en mouvement), on prend en compte la résistance la plus importante compatible avec le seul critère de résistance du matériau (sans préjuger de la distribution des contraintes). Il y a certitude d'instabilité si la puissance des efforts extérieurs est supérieure à la puissance maximale des efforts résistants (condition d'instabilité), et présomption de stabilité si, quel que soit le mécanisme envisagé, la puissance des efforts extérieurs reste inférieure ou égale à la puissance maximale des efforts résistants (il ne peut $y$ avoir que présomption ne serait-ce que parce que l'on n'a pas, sauf exception, la certitude d'avoir envisagé le mécanisme le plus défavorable).

Étudions le cas, on ne peut plus élémentaire, représenté sur la figure $n^{\circ} 1$; le bloc ABCD repose sur un massif semi-infini; le long de CD, la résistance est exprimée au moyen du critère de Coulomb:

$$
|\tau| \leq \sigma \cdot \tan (\varphi)+\mathrm{c}
$$

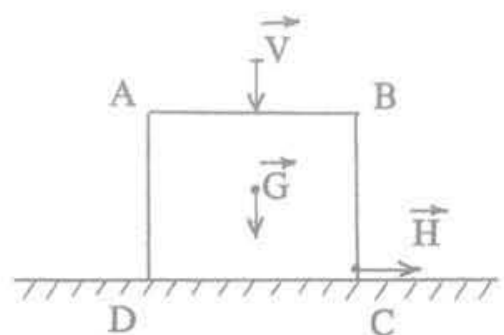

Équilibre d'un bloc reposant sur une surface horizontale.

Equilibrium of a block lying on an horizontal surface.

L'écriture usuelle de l'équilibre du bloc, soumis à son poids $\mathrm{G}$, à une force verticale $\mathrm{V}$ et à une force horizontale $\mathrm{H}$, est :

$$
|H| \leq(G+V) \cdot \tan (\varphi)+c \cdot S
$$

S étant la surface de contact.

Dans ce cas très simple, la méthode cinématique n'apporte rien, mais l'objet est ici de la comprendre,

On applique au bloc $\mathrm{ABCD}$ une vitesse v présentant un angle $\alpha$ avec l'horizontale. La puissance de $\mathrm{G}, \mathrm{V}$ et $\mathrm{H}$ est d'écriture aisée :

$P(G)=-G \cdot v \cdot \sin (\alpha)$

$\mathrm{P}(\mathrm{V})=-\mathrm{V} \cdot \mathrm{V} \cdot \sin (\alpha)$

$\mathrm{P}(\mathrm{H})=\mathrm{H} \cdot \mathrm{v} \cdot \cos (\alpha)$

Venons-en à la résistance le long de la surface de rupture; sur la figure 2a, on envisage $\alpha<\varphi$; le long de la surface de rupture envisagée $C D$, la contrainte prise le long de la limite du critère de résistance $(\sigma$, $\tau=\sigma \cdot \tan (\varphi)+c)$ sur une partie élémentaire de surface dS fournit la puissance :

$$
\mathrm{dP}=\mathrm{dS} \cdot[\sigma \cdot \sin (\alpha)-\tau \cdot \cos (\alpha)] . \mathrm{v}
$$

soit, pour la valeur la plus résistante de $\tau$ compatible avec $\sigma$ :

$$
\mathrm{dP}=\mathrm{dS} \cdot[\sigma \cdot \sin (\alpha-\varphi) / \cos (\varphi)-\mathrm{c} \cos (\alpha)], v
$$

$\sin (\alpha-\varphi)<0$ puisque $\alpha<\varphi$; en faisant tendre $\sigma$ vers l'infini, à la recherche de la contrainte la plus résistante compatible avec le critère (détermination de l'effort résistant maximal), on obtient un effet résistant infini. $\mathrm{Si} \alpha$ est choisi inférieur à $\varphi$, le mécanisme n'est pas pertinent. 
Sur la figure $2 \mathrm{~b}$, on envisage $\alpha \geq \varphi$; la contrainte du domaine de résistance la plus stabilisatrice est alors

$$
\sigma=-c / \tan (\varphi)
$$

elle apporte :

$$
\mathrm{dP}=-\mathrm{dS} . \mathrm{c} . \mathrm{v}, \sin (\alpha) / \tan (\varphi)
$$

soit $\mathrm{P}=-$ S.C.v. $\sin (\alpha) / \tan (\varphi)$

La somme des puissances virtuelles est alors :

$W=H \cdot v \cdot \cos (\alpha)-(G+V) \cdot v \cdot \sin (\alpha)-S \cdot c \cdot v \cdot \sin (\alpha) / \tan (\varphi)$

Si $W$ est positive pour au moins une valeur de $\alpha$, l'équilibre est instable.

$$
\mathrm{W}=\mathrm{v} \cdot \cos (\alpha) \cdot[\mathrm{H}-(\mathrm{G}+\mathrm{V}+\mathrm{S} \cdot \mathrm{c} / \tan (\varphi)) \cdot \tan (\alpha)]
$$

La valeur limite de $H$ compatible avec l'équilibre est, en fonction de $\alpha(\geq \varphi)$ :

$$
\text { Hlim }=(G+V+S \cdot c / t a n(\varphi)) \cdot \tan (\alpha)
$$

La plus petite valeur de Hlim est donc $(G+V) \cdot \tan (\varphi)$ + S.c ; elle est obtenue pour $\alpha=\varphi$.
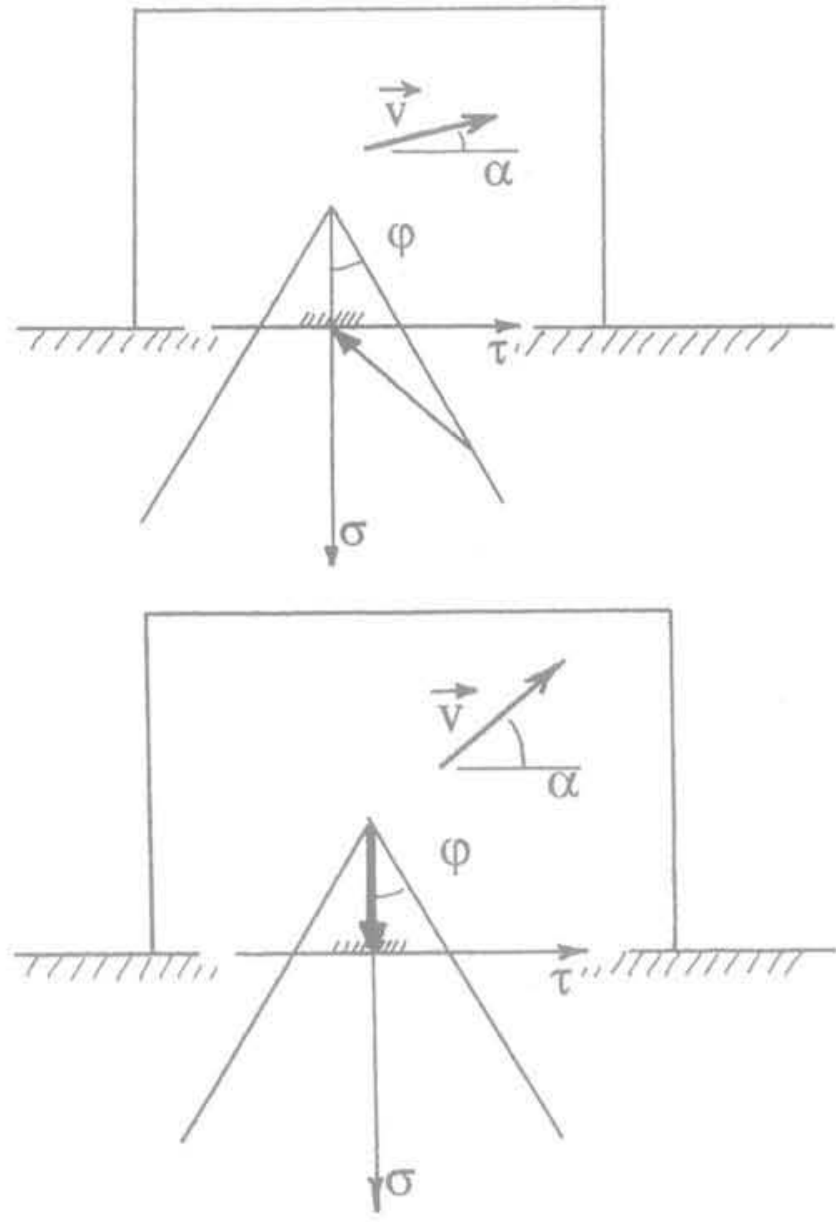

FG. 2 Méthode cinématique. Cinematic method.

La théorie du calcul à la rupture conduit en fait à ne se préoccuper que du cas $\alpha=\varphi$, sans passer par les détours précédents, qui n'ont pour objet que de faire percevoir cette règle à un lecteur non familiarisé avec la méthode cinématique (on trouve la démonstration de cette règle dans l'ouvrage de Salençon).

Un mécanisme constitué d'un seul bloc est tel que la vitesse virtuelle présente en tout point de la surface de discontinuité de vitesse (surface de rupture) un angle égal à $\varphi$. Si le mécanisme comporte plusieurs blocs animés de vitesses virtuelles différentes, le long de la surface de discontinuité séparant deux blocs (ou un bloc et la partie immobile du massif), la différence de vitesses présente un angle égal à $\varphi$ avec cette surface.

La figure 3 représente un mécanisme de rupture dans le cas de l'étude de stabilité d'un talus en sol homogène et isotrope : on envisage une rotation autour du point $\mathrm{O}$, et la ligne directrice, le long de laquelle la vitesse virtuelle présente un angle $\varphi$ avec la tangente, est un arc de spirale logarithmique d'équation $\mathrm{R}=\mathrm{R}_{0} \cdot \mathrm{e}^{\theta \tan (\varphi)}$; contrairement à l'approche classique au moyen de cercles, qui exige de recourir à un artifice d'évaluation des contraintes normales pour disposer de valeurs de résistance (méthodes de tranches), la méthode cinématique n'appelle aucune hypothèse complémentaire ; on note que l'on retrouve la spirale de Rendulic, schéma selon lequel l'apport de $\varphi$ n'intervient pas dans l'écriture de l'équilibre en moment (forces élémentaires portées par des rayons). Les artifices associés aux méthodes tranches introduisent une incertitude ; nous avons un jour établi à l'aide de la méthode cinématique un abaque relatif à la stabilité des talus, que nous avons comparé à l'abaque de TaylorBiarez, établi pour sa part au moyen d'une méthode de tranches ; les écarts n'excèdent guère $5 \%$, en plus ou en moins... sachant que la méthode cinématique ne peut pas conduire à un résultat conservatif (approche à coup sûr par l'extérieur de la sécurité).

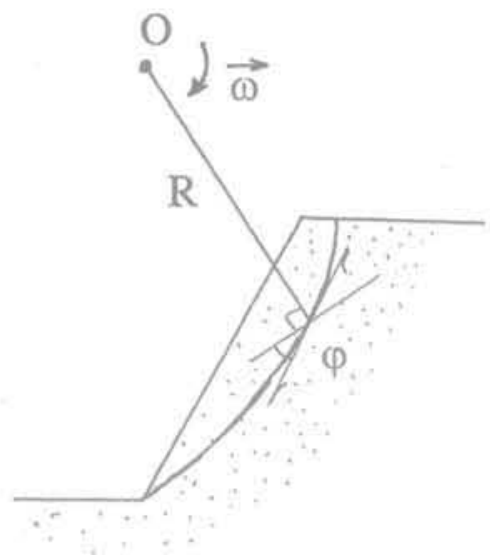

Méthode cinématique: cas d'un talus. Cinematic method in the case of a slope.

Abordons maintenant l'étude du « Coin de Coulomb » en sol homogène. La figure 4a traduit l'écriture usuelle, qui conduit à la résolution des équations:

$$
\begin{gathered}
E \cdot \cos (\delta)=R \cdot \sin (\beta-\varphi) \\
G=R \cdot \cos (\beta-\varphi)+E \cdot \sin (\delta)
\end{gathered}
$$

La figure $4 \mathrm{~b}$ correspond à la méthode cinématique, qui conduit à l'équation:

$$
G \cdot \sin (\beta-\varphi) \cdot v=E \cdot \cos (\beta-\varphi-\delta), v
$$

On obtient par les deux approches la même valeur de $E$ en fonction de $\beta$, dont il reste à déterminer la valeur la plus défavorable. L'approche cinématique parait plus simple, mais la simplicité serait comparable si, dans l'approche classique, on effectuait la projection sur la perpendiculaire à $\mathrm{R}$ au lieu de projeter sur la verticale et l'horizontale (équation unique, la même au facteur v près). 
a :

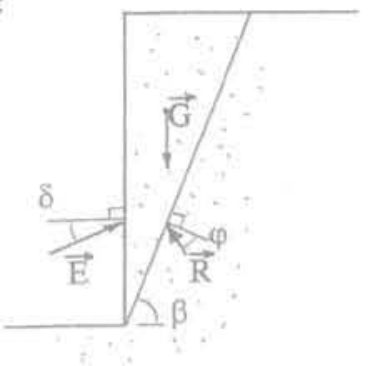

Fig. 4 b :

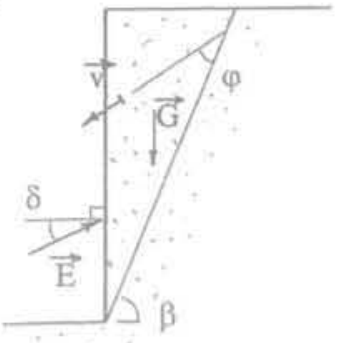

Coin de Coulomb en sol homogène. Coulomb's wedge, homogeneous soil?

Passons au cas du sol multicouche, pour lequel l'avantage de la méthode cinématique devient évident. La figure 5a correspond à l'approche classique : on ne sait résoudre le problème qu'en introduisant des hypothèses plus ou moins arbitraires quant aux valeurs respectives attribuées à $R_{1}, R_{2}$ et $R_{3}$. La figure 5 b traduit l'approche cinématique : le choix de l'angle $\gamma$ figeant l'orientation de la vitesse $v$ détermine les angles $\beta$. $\left(=\gamma+\varphi_{1}\right), \beta_{2}$ et $\beta_{3}$; la géométrie est alors figèe, et $G$ est facile à calculer ; on n'a besoin de résoudre qu'une seule équation, sans qu'il soit nécessaire de faire appel à une quelconque hypothèse complémentaire ; il reste évidemment à déterminer la valeur la plus défavorable de l'angle $\gamma$.

\section{9}

\section{Critère de Coulomb tronqué en traction}

La résistance d'un sol à des contraintes de traction est un domaine que l'on n'explore qu'exceptionnellement lorsqu'on mesure sa résistance. Et si une résistance en traction existe, elle présente généralement un caractère de fragilité et il n'est donc pas raisonnable de la prendre en compte dans la justification de stabilité d'un ouvrage courant. On peut alors utiliser un «critère tronqué en traction s, $_{\text {, }}$ que schématisé sur la figure 6 ; le domaine de résistance du matériau est constitué d'une partie du cercle de Mohr correspondant à la compression simple et de deux demi-droites tangentes, de pentes égales à $\pm \tan (\varphi)$, étant entendu que certains sols présentent d'autres critères de résistance (par exemple critère parabolique).

a :

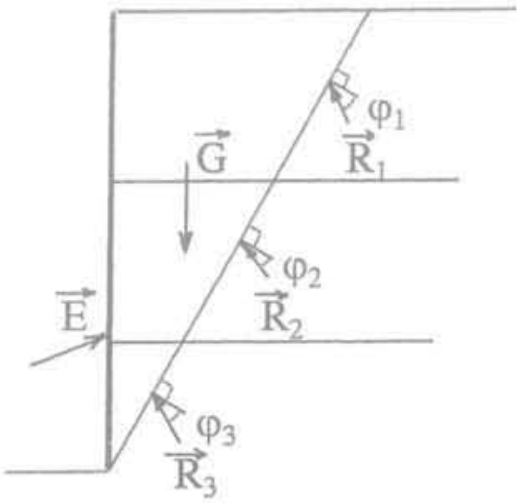

b :

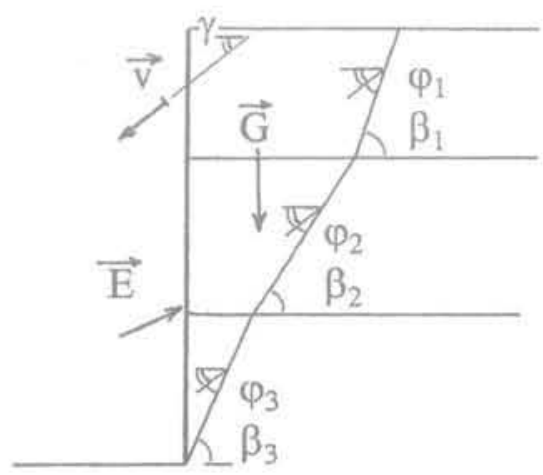




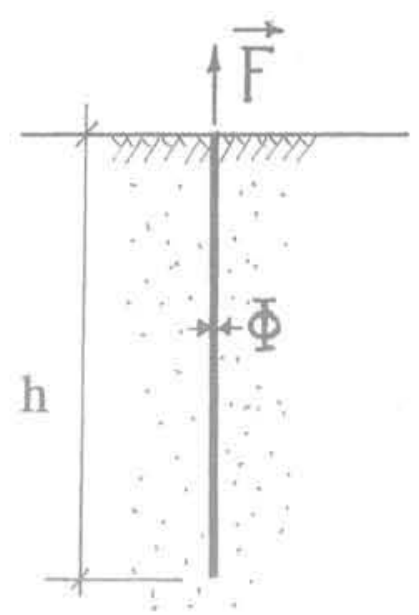

FIG. 7

Ancrage isolé.

Isolated anchor.

On applique une force axiale F. La rupture peut être gouvernée par la résistance de l'armature, le frottement latéral limite, et/ou le sol (résistance au cisaillement et poids volumique) ; on né se préoccupera pas ici de la résistance de l'armature.

La défaillance supposée liée au seul frottement latéral limite (supposé constant le long de l'ancrage) correspond à une force limite:

$$
\mathrm{F}_{\text {limi }}=\pi \cdot \phi \cdot h \cdot \mathrm{q}_{\mathrm{s}}
$$

Note: une application rigoureuse de la méthode cinématique nécessite d'écrire d'abord une équation en puissances; lorsque $v$ apparait en facteur dans les deux termes de l'équation, on peut omettre son écriture, comme nous le ferons tout au long du présent article (sous réserve bien entendu que le signe de v soit pertinent).

Le problème est axisymétrique, et pour traiter une rupture du sol, on envisage une vitesse virtuelle vertjcale ascendante. Une surface axisymétrique qui présente en tout point un angle égal à $\varphi$ avec la verticale est une portion de cône d'axe vertical confondu avec celui de l'ancrage.

La défaillance supposée liée au seul angle de frottement interne correspond ainsi à un tronc de cône d'angle au sommet égal à $\varphi$, et de petite base correspondant à la section de l'extrémité inférieure du forage : dans la pratique, on néglige le plus souvent le diamètre du forage pour apprécier ce volume, ce qui conduit à :

$$
\mathrm{F}_{\lim 2}=\pi \cdot h^{3} \cdot \gamma \cdot \tan ^{2}(\varphi) / 3
$$

$\gamma$ étant le poids volumique «utile ) (déjaugé dans le cas de la sous-pression).

La figure 8 présente l'évolution de $F_{\text {inj }}$ et $F_{\text {ind }^{2}}$ en fonction de h. On constate que pour de faibles valeurs de $h$, c'est le soulèvement d'un cône de sol qui conduit à la plus faible résistance, tandis que pour de grandes valeurs de h, c'est le frottement latéral.

Les deux modes de rupture envisagés jusqu'ici sont en fait deux cas particuliers $(\mathrm{a}=0$ et $\mathrm{a}=\mathrm{h}$ ) du mécanisme schématisé sur la figure 9 , conditionné pour partie par le frottement latéral et pour le reste par la résistance du sol.

Il convient donc de déterminer la valeur la plus défavorable de la longueur a, la résistance limite étant la somme du poids du cône de hauteur $(\mathrm{h}-\mathrm{a})$ et du frottement latéral limite sur la longueur a :

$$
\mathrm{F}_{\mathrm{hm} 3}=\min \left[\pi \cdot \phi \cdot q_{\mathrm{s}} \cdot \mathrm{a}+\pi \cdot(\mathrm{h}-\mathrm{a})^{3} \cdot \gamma \cdot \tan ^{2}(\varphi) / 3\right]
$$

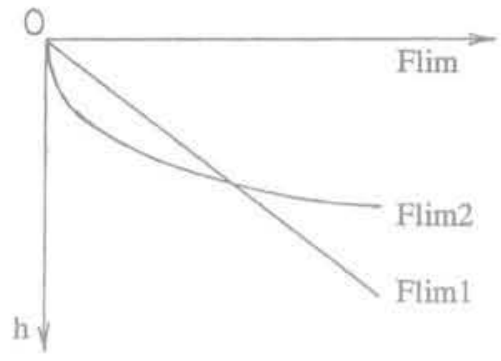

FiG.8. Résistance pour des mécanismes simples. Strength for simple mechanisms.

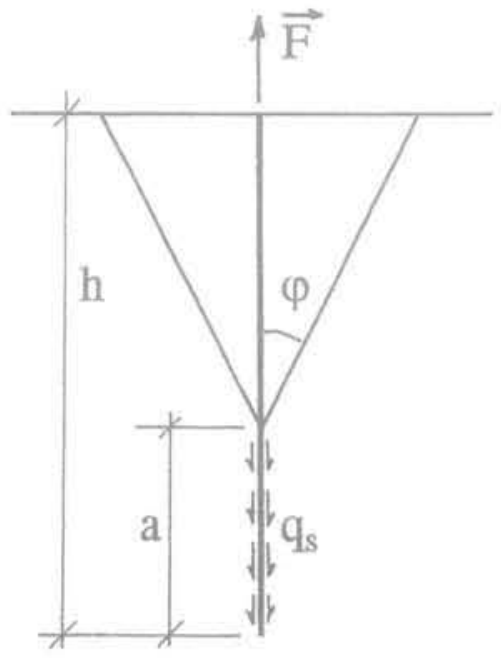

FIG.9 Mécanisme mixte. Mixed mechanism.

La figure $\mathrm{n}^{\circ} 10$ montre l'évolution de $\mathrm{F}_{\mathrm{lum} 3}$ en fonction de $\mathrm{h}$; les courbes précédentes y sont rappelées en pointillés.

Pour $\mathrm{h}<\mathrm{h}$, le sommet du cône est confondu avec l'extrémité de l'ancrage, et pour $h>h_{c}$ le cone est de hauteur égale à h.

La longueur critique $h_{\text {cr }}$ est solution de:

$$
\phi \cdot q_{5}=\gamma \cdot\left(h_{t r} \cdot \tan (\varphi)\right)^{2}
$$

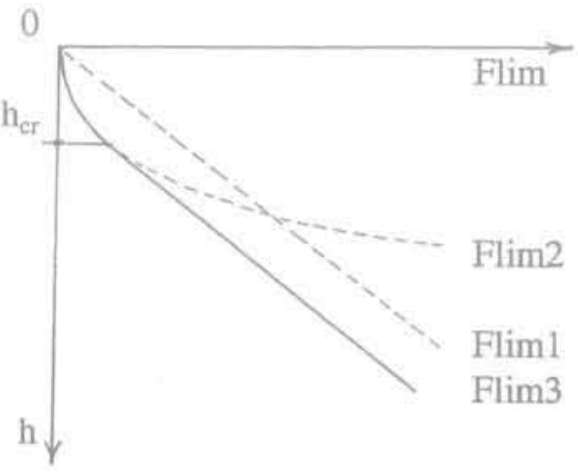

FIG. 10 Résistance pour le mécanisme mixte. Strength for mixed mechanism.

Nous avons négligé, pour l'appréciation du volume, le diamètre de l'ancrage; les formulations peuvent aisément être adaptées pour les cas où le diamètre et le poids volumique de l'ancrage sont susceptibles de jouer un rôle significatif. 


\section{Sol stratifié}

Les strates sont supposées horizontales. Le cône précédent devient un ensemble de troncs de cônes comme l'indique la figure 11, l'angle de cône intéressant chaque sol étant égal à son angle de frottement interne.

Contrairement au cas du sol homogène, la hauteur critique n'est pas toujours unique ; on se reportera utilement à l'annexe 1 qui présente un exemple de conditions conduisant à plusieurs valeurs de $h_{c}$; pour la justification d'un ouvrage, il faut bien entendu déterminer le cas le plus défavorable.

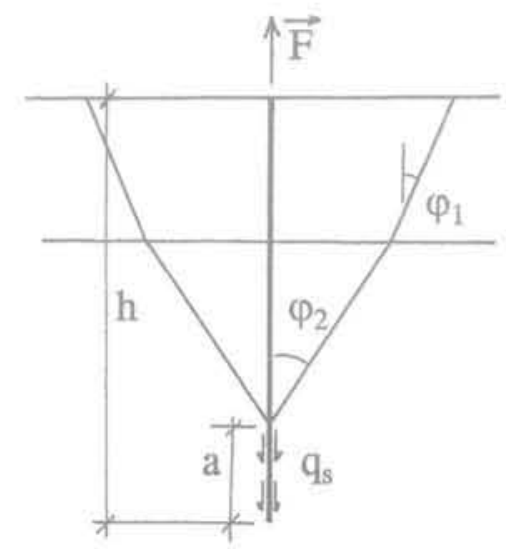

FiG.11 Mécanisme de rupture dans un massif bicouche.

Two-layer soil mass, failure mechanism.

\section{Ancrage isolé, cohésion non nulle}

\section{1}

\section{Critère de Coulomb " complet »}

Le théorème des états correspondants permet de substituer au problème celui d'un sol purement frottant et soumis à une pression extérieure de valeur $c / \tan (\varphi)$. Voir la figure $n^{\circ} 12$ a pour un sol homogène.

Dans le cas d'un massif homogène, $\mathrm{F}_{\mathrm{lim} 2}$ est alors ;

$$
F_{\text {lim2 } 2}=\pi \cdot h^{3} \cdot \gamma \cdot \tan ^{2}(\varphi) / 3+\pi \cdot h^{2} \cdot \tan (\varphi) \cdot c
$$

et $\mathrm{F}_{\text {lim3 }}$ a pour expression :

$$
\begin{gathered}
\mathrm{F}_{\mathrm{lim} 3}=\min \left[\pi \cdot \phi \cdot \mathrm{q}_{\mathrm{l}} \cdot \mathrm{a}+\pi \cdot(\mathrm{h}-\mathrm{a})^{3} \cdot \gamma \cdot \tan ^{2}(\varphi) / 3\right. \\
\left.+\pi \cdot(\mathrm{h}-\mathrm{a})^{2} \cdot \tan (\varphi) \cdot c\right] \text { en fonction de a }
\end{gathered}
$$

On retrouve la notion de $\mathrm{h}_{\mathrm{cr}}$ solution cette fois de :

$$
\phi \cdot q_{\mathrm{s}}=\gamma \cdot\left(\mathrm{h}_{\mathrm{cr}} \cdot \tan (\varphi)\right)^{2}+2 \cdot c \cdot \mathrm{h}_{\mathrm{cr}} \cdot \tan (\varphi)
$$

Dans le cas d'un sol stratifié, la prise en compte de la cohésion au moyen du théorème des états correspondants conduit à des mécanismes dont la géométrie est conditionnée par les angles de frottement interne, avec sur chaque interface des pressions correspondant aux caractéristiques de chaque sol, selon les indications de la figure $12 \mathrm{~b}$.

\section{2}

\section{Critère de Coulomb tronqué en traction}

La prise en compte de ce critère dans un calcul à la rupture au moyen de la méthode cinématique conduit à envisager des surfaces de rupture moins évidentes. Paradoxalement, il est alors plus facile d'aborder un ensemble d'ancrages répartis selon une trame qu'un ancrage isolé. Nous allons donc traiter le cas d'une trame avant de revenir à l'ancrage isolé.

\section{5}

\section{Ancrages répartis selon une trame}

\section{1}

\section{Deux ancrages voisins ne doivent pas mobiliser un même volume de sol}

Bien que l'on puisse envisager des répartitions complexes (par exemple lorsque le radier est simultanément équilibré par des charges de poteaux), il est souvent raisonnable d'associer à chaque ancrage un volume limité par des plans verticaux dont la trace dans le plan horizontal définit des « cellules » avec un ancrage au centre de chaque cellule et, pour des sols sans cohésion, un schéma de rupture selon des cônes, comme cela est présenté sur la figure 13 pour un sol homogène. a :

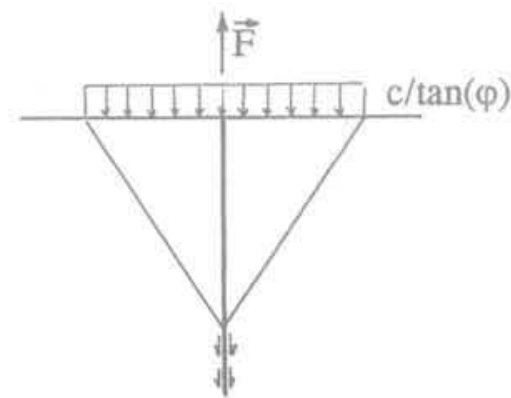

b:

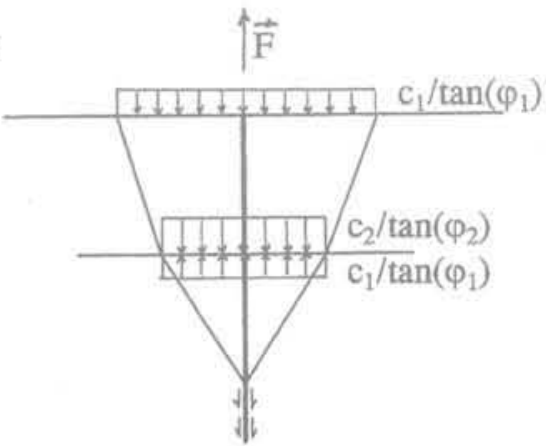

FIG. 12 Prise en compte de la cohésion : critère de Coulomb « complet $).$ Accounting for the cohesion: use of a complete Coulomb's criterion. 
vue en plan

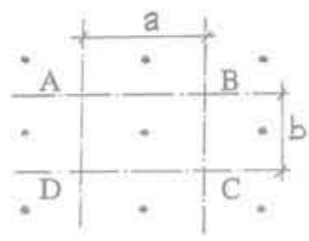

schéma d'une * cellule »

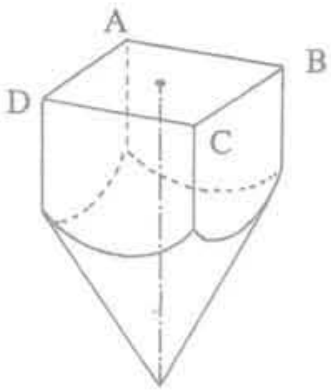

FiG. 13 Volume associé à un ancrage long au sein d'une trame.

Long anchor, within a mesh, associated volume.

Il est intéressant de remarquer qu'en sol homogène, au-delà d'une certaine longueur, une variation de position du sommet du cône dh, pour une trame rectangulaire $\mathrm{a} \times \mathrm{b}$, se traduit par une variation de volume dh.a.b, et donc si $\mathrm{q}_{\mathrm{s}}>\mathrm{a} \cdot \mathrm{b} \cdot \gamma /(\pi \cdot \phi)$, l'extrémité de l'ancrage impose la position du sommet du cône (des précautions s'imposent pour étendre cette remarque à des sols stratifiés).

Pour un sol purement frottant, le calcul du volume de sol associé à un ancrage relève usuellement dans la pratique de méthodes numériques, ou d'un recours à des abaques (TA95). On peut néanmoins signaler que ce volume peut aussi être déterminé au moyen d'une intégration analytique. L'annexe 2 présente une telle intégration dans les cas simples d'un sol homogène et d'une trame carrée ou triangulaire.

\section{2}

\section{Motif de la trame et axisymétrie}

Pour un sol purement frottant, avec une trame assez serrée et des ancrages assez longs pour que les cônes voisins soient sécants au-dessous de la sous-face du radier, le frottement latéral étant supposé surabondant, on peut aisément définir l'épaisseur moyenne du massif de sol solidaire des ancrages.

Lè calcul analytique est présenté dans l'annexe 2, pour une trame carrée, puis pour une trame triangulaire.

L'erreur commise en substituant au motif carré ou hexagonal un motif circulaire dont la section horizontale a la même aire est petite devant les incertitudes relatives à la géométrie et à la résistance au cisaillement des sols. Nous admettrons, sans être en mesure de le démontrer, que l'erreur reste acceptable dans le cas du critère de Coulomb tronqué en traction; le modèle axisymétrique permet en effet de se ramener à une géométrie bidimensionnelle et de déterminer sans difficulté majeure un mécanisme que l'on peut présumer être le plus défavorable.

\section{3}

\section{Critère de Coulomb tronqué en traction, sol homogène}

Le vecteur vitesse virtuelle est vertical. La surface de rupture que l'on cherche est axisymétrique. La

figure 14 représente, en un point de la surface de rupture, le domaine de résistance. Si $\alpha$ est inférieur à $\varphi$, il existe dans le domaine de résistance des contraintes susceptibles d'équilibrer n'importe quel chargement : pour que la surface de discontinuité soit pertinente, il faut donc respecter en tout point $\alpha \geq \varphi$. Pour $\alpha \geq \varphi$, la contrainte résistante la plus favorable possible a pour composante parallèle à l'axe de l'ancrage (qui porte la vitesse virtuelle) :

$$
\text { c. }(1-\sin (\alpha)) / \tan (\pi / 4-\varphi / 2) \text {. }
$$

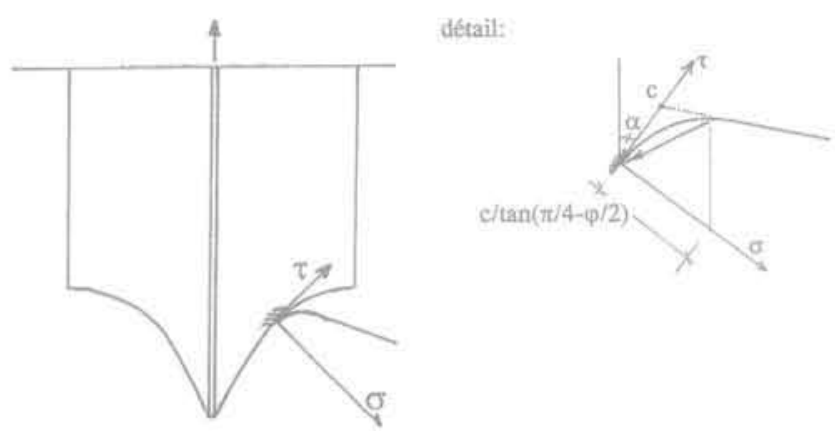

FiG. 14 Domaine de résistance en un point de la surface de rupture. Strength domain in a point of the failure zone.

Le volume du bloc solidaire de l'ancrage dans le mécanisme de rupture peut être comparé à celui du cylindre dont la base passe par l'extrémité de l'ancrage. Dans le cas du cylindre, le volume est égal à S.L, et la cohésion n'apporte aucune contribution à la résistance; son poids est un majorant de la résistance cherchée. La surface que l'on cherche génère un moindre volume, ce qui réduit le rôle stabilisateur des forces de pesanteur, mais simultanément la cohésion contribue à la résistance. Notre propos est de déterminer la surface à laquelle correspond la plus petite résistance, donc celle qui minimise la somme des effets de la pesanteur et de la cohésion.

Pour ce faire, on peut se ramener à une détermination point par point de la pente : on envisage des tranches horizontales minces (Fig. 15) d'épaisseur $\mathrm{dy}=\mathrm{dr} / \tan (\alpha)$. La pente locale de la courbe ne conditionne que les contributions de la cohésion et de la

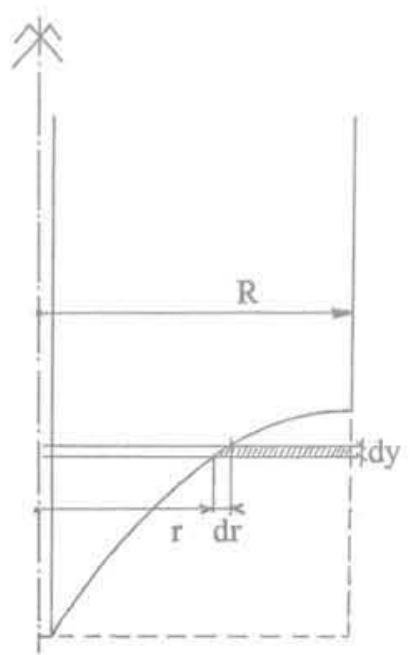

FIG.15 Tranche de calcul. Slice used in the calculation. 
pesanteur associées à cette tranche, la recherche du minimum précité se ramène ainsi à la recherche des minima locaux.

Envisageons une tranche d'épaisseur dy : la surface de rupture la coupe selon un cercle de rayon $r$ au niveau inférieur et $\mathrm{r}+\mathrm{dr}$ au niveau supérieur. On doit vérifier $\mathrm{dy} \leq \operatorname{dr} / \tan (\varphi)$,

La contribution locale de la cohésion s'exprime, pour une vitesse virtuelle unité :

$$
\mathrm{dF}=2 . \pi . r . c,\left(\left(d r^{2}+d y^{2}\right)^{0.5}-d r\right) / \tan (\pi / 4-\varphi / 2)
$$

La variation de contribution de la pesanteur (en référence au cylindre) est alors, $R$ étant le rayon de la cellule axisymétrique :

$$
\mathrm{dP}=-\pi \cdot\left(\mathrm{R}^{2}-r^{2}\right) \cdot \gamma \cdot d y
$$

On cherche dy tel que $\mathrm{dF}+\mathrm{dP}$ soit minimal ; on dérive par rapport à dy la fonction $\mathrm{dF}+\mathrm{dP}$, dérivée qui s'annule pour:

$$
\mathrm{dy}=\mathrm{dr} \cdot \gamma \cdot\left(\mathrm{R}^{2}-\mathrm{r}^{2}\right) /\left[4 \cdot \mathrm{r}^{2} \cdot \mathrm{c}^{2} / \tan ^{2}(\pi / 4-\varphi / 2)-\gamma^{2} \cdot\left(\mathrm{R}^{2}-r^{2}\right)^{2}\right]^{25}
$$

Si cette équation conduit à dy $>d r / \tan (\varphi)$, il convient bien entendu de retenir $d y=d r / \tan (\varphi)$. En pratique, il existe une valeur $r$, de $r$ telle que, pour $r<r_{1}, d y=d r / \tan (\varphi)$, dy découlant de l'expression précédente si $r>r_{1}$ :

$$
r_{1}=-c \cdot(1+\sin (\varphi)) / \gamma+\left[(c \cdot(1+\sin (\varphi)) / \gamma)^{2}+R^{2}\right]^{0,5}
$$

On est donc en mesure de déterminer point par point la pente de la ligne directrice de la surface de rupture, et de calculer la différence entre la force stabilisatrice et celle qu'apporterait le poids d'un cylindre de hauteur égale à celle de l'ancrage. Ce calcul est réalisé au moyen d'un programme rédigé en QBASIC et listé en annexe (programme ANCRRAD, susceptible d'effectuer aussi des calculs liés aux considérations présentées ci-après; on peut noter que le programme prend en compte le rayon de l'inclusion, en lui attribuant le même poids volumique qu'au sol).

On peut illustrer les résultats que l'on obtient par une représentation graphique correspondant à des valeurs numériques ; on choisit $\gamma=10 \mathrm{kN} / \mathrm{m}^{3}, \varphi=30^{\circ}$,

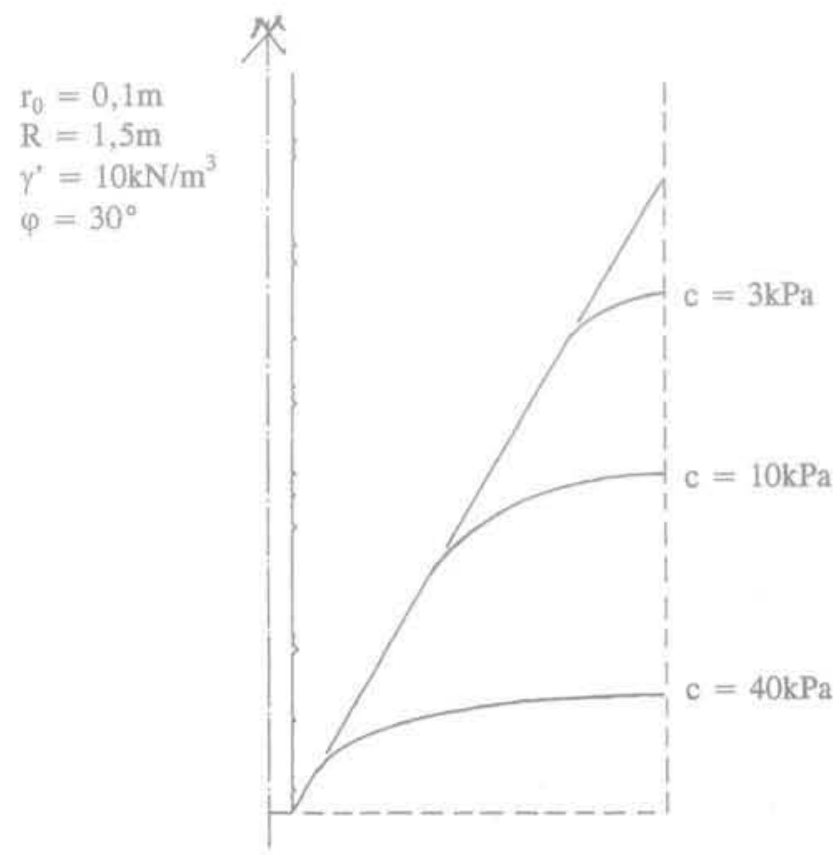

FIG.16 Exemple de surfaces de rupture, cellule axisymétrique,

Examples of failure surfaces, axisymetric cell. $r_{0}=0,1 \mathrm{~m}$ et $\mathrm{R}=1,5 \mathrm{~m}$. La figure 16 montre la surface de rupture obtenue avec $\mathrm{c}=3 \mathrm{kPa}, \mathrm{c}=10 \mathrm{kPa}$ et $\mathrm{c}=40 \mathrm{kPa}$. La figure 17 montre, en fonction de c, la différence entre la longueur des ancrages et l'épaisseur d'un matelas de sol dont le poids équivaut à la force stabilisatrice que peuvent mobiliser les ancrages (il ne s'agit pas du volume correspondant au mécanisme de rupture, car la résistance du sol le long de la surface de rupture est prise en compte). On constate qu'une cohésion modeste apporte une contribution non négligeable.

\section{4}

\section{Sol bicouche}

Contrairement à ce que nous venons de voir pour un sol homogène, si la surface de rupture est conditionnée par deux strates, la discrétisation en tranches horizontales r'autorise pas l'étude séparée de chaque tranche : en partant de la base de l'ancrage, la pente de la surface de rupture conditionne les contributions locales de la cohésion et de la pesanteur, et conditionne aussi le rayon du cercle d'intersection éventuelle de la surface de rupture avec l'interface entre les deux strates; il ne suffit d'ailleurs pas de constater que la surface de rupture correspondant aux caractéristiques de la seule strate inférieure ne ( remonte » pas assez haut pour atteindre l'interface pour être certain que la géométrie la plus défavorable n'intéresse pas les deux sols.

Un moyen de détermination de la surface de rupture, certes laborieux (à moins de l'automatiser), est le suivant : on envisage diverses valeurs de dénivellation entre l'extrémité de l'ancrage et le point où la ligne directrice de la surface de rupture atteint la limite du modèle ; entre ces deux extrémités, on procède à une optimisation de la directrice (algorithme analogue à

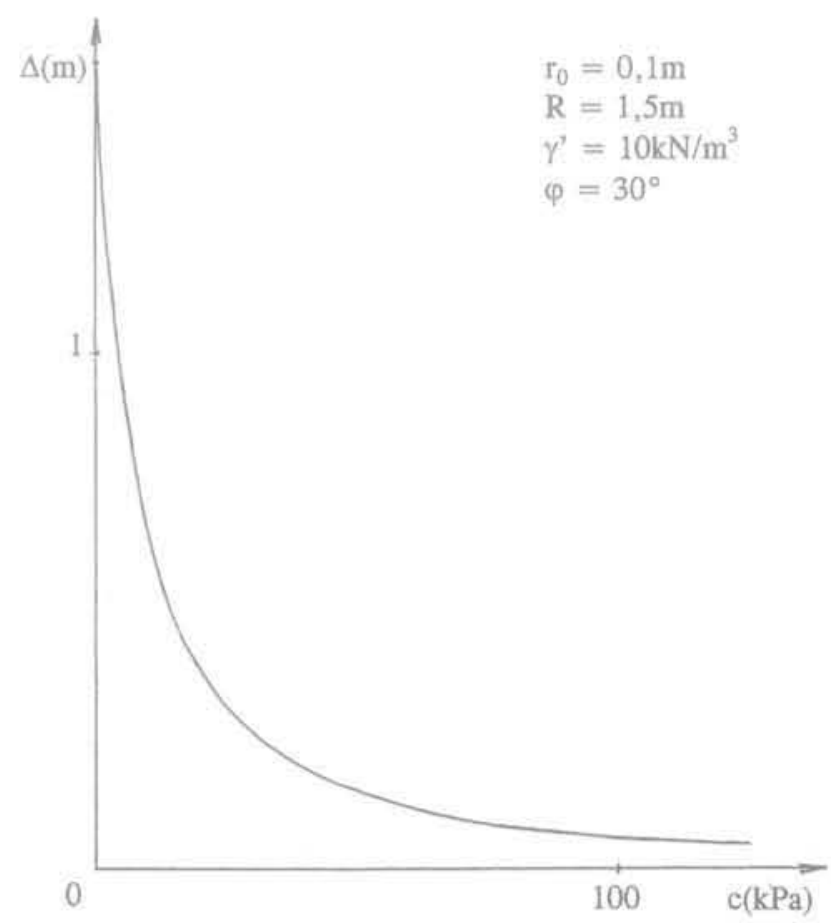

FIG.17 Différence entre longueur des ancrages et épaisseur d'un matelas équivalent. Difference between the anchor length and the thickness of an equivalent matress of soil. 
celui mis en œuvre dans le programme ANCRCOUR listé en annexe que nous avons utilisé pour l'étude des ancrages isolés) ; on tâtonne sur la dénivellation à la recherche de la valeur la plus défavorable.

Si la cohésion est modeste, on peut en pratique être conservatif, la négliger, et se ramener ainsi à des considérations simples...

\section{6}

\section{Retour sur le cas de l'ancrage isolé}

La détermination de la géométrie de la surface de rupture est aisée pour un rayon de modèle donné, comme nous l'avons vu ci-dessus. Mais, dans le cas d'un ancrage isolé, le rayon du modèle n'est pas une donnée du problème.

On peut suivre une première démarche. On admet que le frottement latéral ne conditionne pas la rupture; pour une longueur donnée d'un ancrage, on envisage divers rayons d'émergence de la surface de rupture; pour chaque rayon d'émergence, un calcul itératif permet d'optimiser la géométrie de la ligne directrice (en discrétisant cette ligne en segments de droite), et de déterminer la résistance associée au rayon d'émergence ; par tâtonnements successifs, on identifie le rayon le plus défavorable (c'est l'objet du programme ANCRCOUR listé en annexe). Pour déterminer si le frottement latéral conditionne ou non la résistance de l'ancrage, il est nécessaire d'effectuer la détermination précédente pour diverses longueurs de l'ancrage, la longueur critique étant celle au-delà de laquelle le mécanisme conduit à une variation plus rapide de la résistance que celle de la résistance associée au seul frottement latéral.

Une deuxième démarche peut être envisagée. On assimile le rayon d'émergence à celui d'un modèle axisymétrique d'ancrage au sein d'une trame; le calcul conduit alors à une dénivellation entre l'extrémité de l'ancrage et l'autre extrémité de la directrice de la surface de rupture ; on cherche par itérations successives le rayon du modèle qui conduit à une dénivellation égale à la longueur de l'ancrage considéré. Cette deuxième approche est beaucoup plus économique en temps de calcul.

La première approche est rigoureuse ; la deuxième ne l'est pas : elle suppose une relation biunivoque entre dénivellation et rayon à l'optimum que nous sommes incapables de démontrer; nous avons seulement constaté, sur un nombre nécessairement límité de combinaisons de paramètres, que les deux démarches aboutissent au même résultat ; comme la deuxième est beaucoup plus rapide, nous l'avons intégrée au programme ANCRRAD, pour traiter les cas de trame et de frottement latéral conduisant à un fonctionnement indépendant des ancrages.

La figure 18 montre, pour un ancrage au sein d'une trame, les géométries des mécanismes de rupture en fonction de la longueur de l'ancrage, le frottement latéral étant surabondant. Pour de faibles longueurs de l'ancrage, les lignes directrices du mécanisme de rupture émergent en surface ; elles sont tangentes à la surface du massif; si le frottement latéral est trop petit, c'est une surface de ce type qui conditionne la résistance. La ligne en pointillés correspond à une longueur en deçà de laquelle l'ancrage fonctionne comme un ancrage isolé ; pour de plus grandes longueurs la géométrie de la surface de rupture ne varie plus qu'en s'approfondissant.

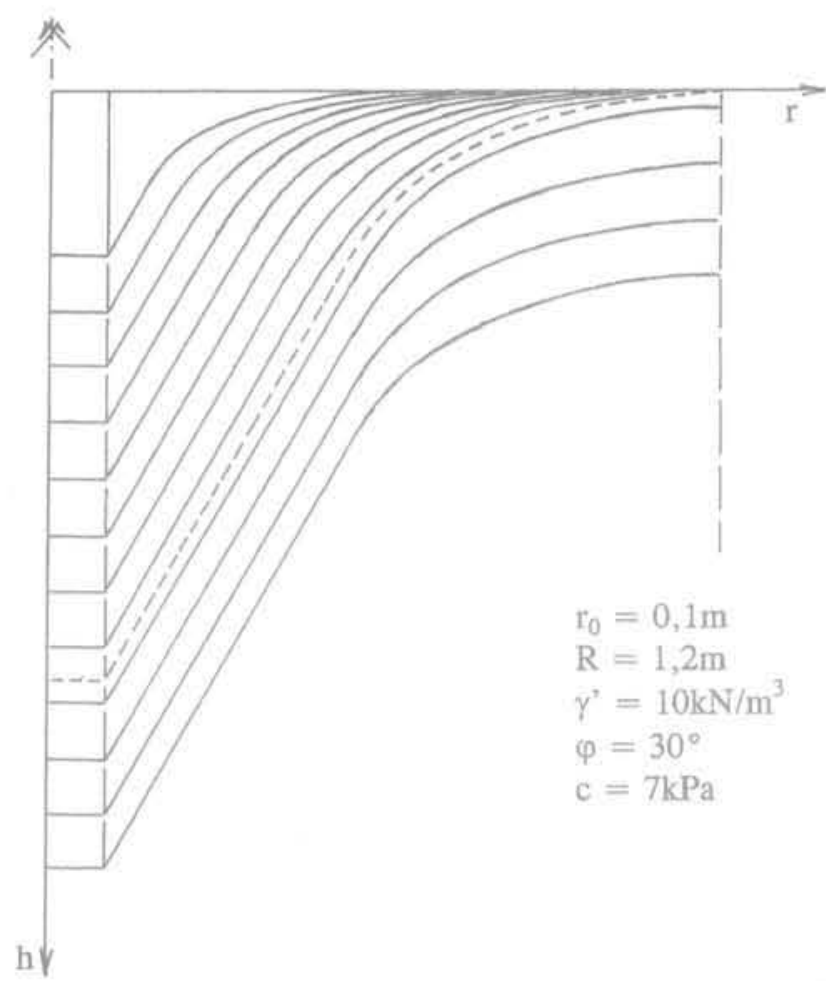

FIG. 18 Géométrie de la rupture en fonction de la longueur d'un ancrage.

Failure shape as a function of the anchor length.

La figure 19 montre, pour un cas particulier, la variation de la résistance disponible en fonction de la longueur de l'ancrage, du frottement latéral limite, et du ravon du modèle axisymétrique. La courbe en trait plein correspond à la résistance d'un ancrage isolé disposant d'un frottement latéral surabondant ; les demidroites en pointillés traduisent un plafonnement de la résistance pour quelques valeurs de frottement latéral; les demi-droites en trait d'axe traduisent un plafonnement de la résistance par le rayon du modèle axisymétrique. On remarque que les demi-droites sont tangentes à la courbe en trait plein. Cest ce fait qui est à l'origine de l'équivalence des deux démarches relatives aux ancrages isolés.

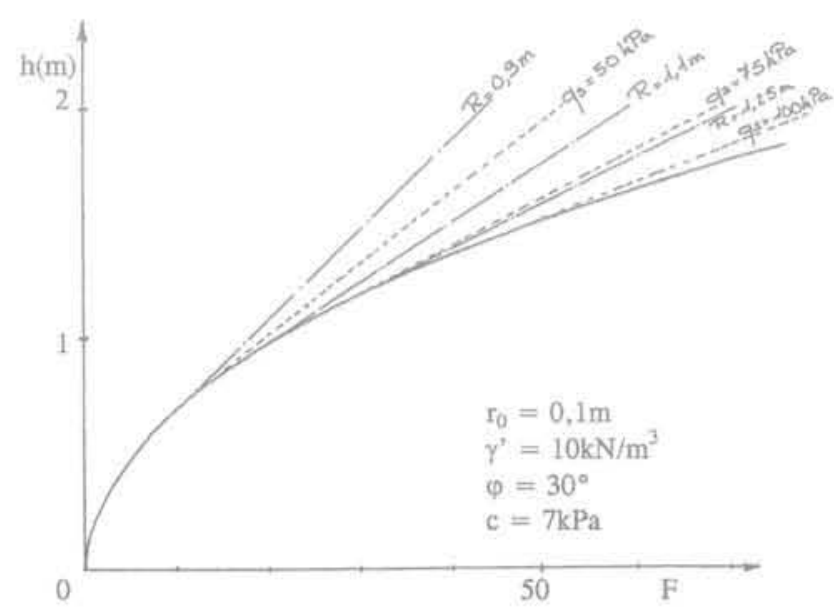

FIG. 19 Traction limite géotechnique. Limit geotechnical tension. 


\section{7}

\section{Variation de la traction le long d'un ancrage}

Nous n'avons jusqu'ici tenu aucun compte de la résistance de l'armature, présumée surabondante. Pour certains ancrages, tels que des pieux en béton armé, on peut souhaiter optimiser le ferraillage, en ne plaçant à chaque niveau que la section d'armature nécessaire. Dans ce paragraphe, nous ne nous préoccuperons toujours pas de marge de sécurité, pour rester sur la physique des phénomènes.

La variation de l'effort de traction entre la tête de l'ancrage et la profondeur z ne peut pas être supérieure à la traction limite (géotechnique) d'un ancrage de longueur z. Au voisinage de la surface, ce n'est donc généralement pas le frottement latéral qui conditionne l'évolution de la traction avec la profondeur.

En pratique, deux approches sont envisageables pour un ancrage dont on a au préalable déterminé la longueur nécessaire :

- si l'on souhaite être rigoureux, on détermine la capacité géotechnique d'un ancrage en fonction de sa longueur z, qui fournit la réduction de traction depuis la tête pour un ancrage plus long (on tient compte bien entendu d'une éventuelle disposition tramée des ancrages) ;

- si l'on veut faire simple en restant conservatif. Pour un ancrage isolé, on considère que la traction augmente à partir de zéro à la base en fonction du frottement latéral, jusqu'à atteindre la traction maximale, qui ne varie plus jusqu'à la tête, et on a ainsi une enveloppe de la résistance en traction nécessaire de la part de l'armature ; pour un ancrage faisant partie d'une trame, on utilise le plus défavorable du frottement latéral et de la section horizontale de la cellule.

La figure 20 présente pour un même ancrage les courbes de traction en fonction de la profondeur selon les deux approches dans un cas particulier. En traits pleins, il s'agit de la détermination précise pour un ancrage isolé et pour le même ancrage au sein d'une

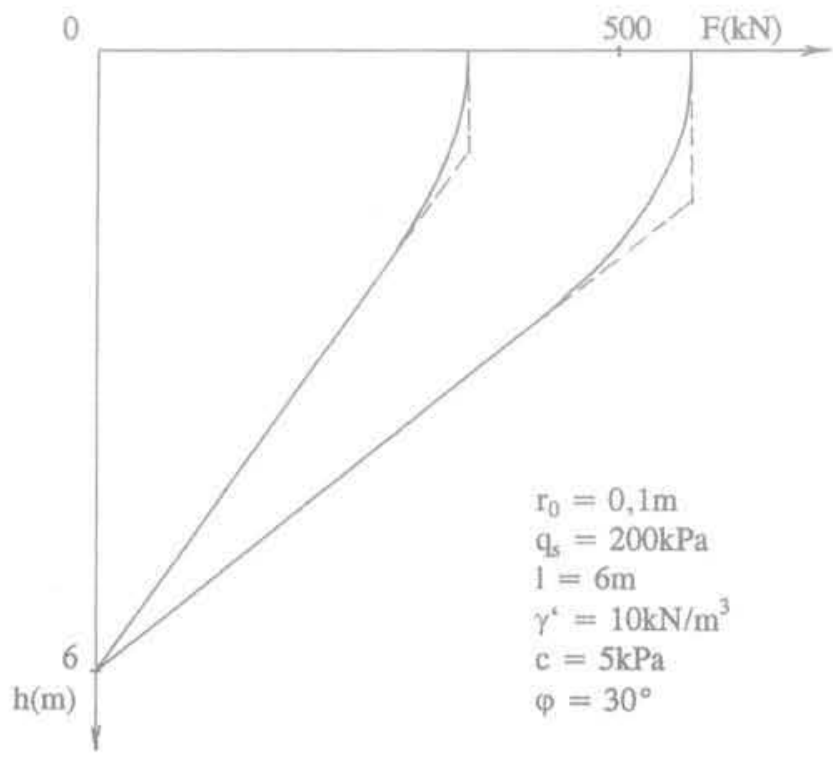

FG. 80 Traction le long de l'armature. Tension along the anchor. trame correspondant à un rayon de modèle axisymétrique égal à $1,5 \mathrm{~m}$; les traits pointillés constituent l'enveloppe obtenue par l'approche simplifiée. L'approche simplifiée ne constitue que rarement une enveloppe pénalisante.

\section{8}

\section{Cas d'un sol purement cohérent}

Notons que le frottement latéral limite ne peut alors pas excéder la cohésion.

Le critère de résistance pris en compte dans la méthode cinématique reste tronqué en traction. La figure 21 présente les schémas de rupture obtenus pour un cas particulier, l'ancrage étant soit isolé, soit dans une trame. On remarque que ces schémas sont très différents de celui que préconisent dans ce cas les TA95, un cóne d'ouverture $45^{\circ}$... (n'oublions pas que le poids n'intervient pas seul, et qu'il faut lui ajouter la contribution de la résistance du sol le long de la surface de rupture).

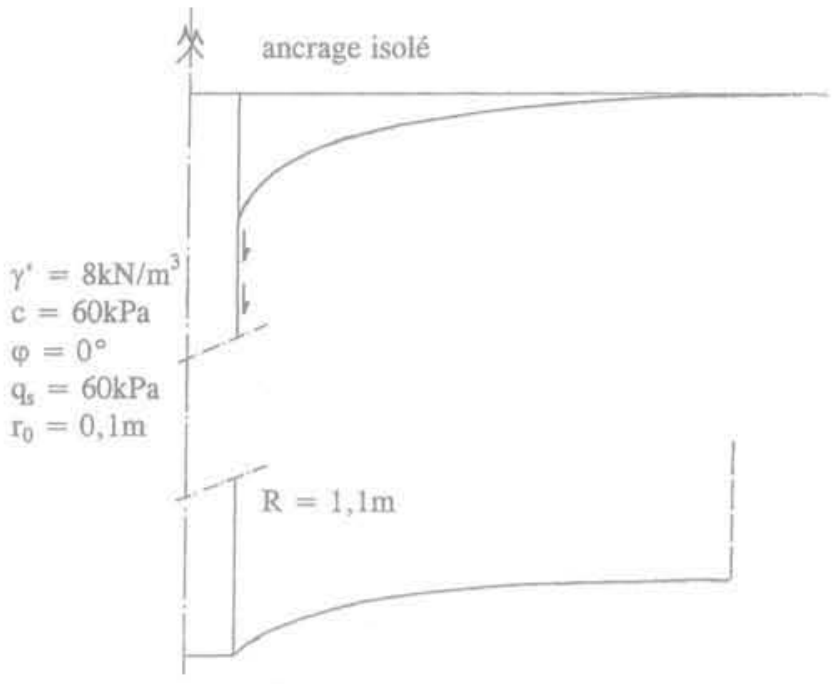

FIG. 21 Schémas de rupture avec $\varphi=0^{\circ}$. Failure sketches with $\varphi=0^{\circ}$.

\section{9}

\section{Incidence de la coupure de résistance en traction}

Si tout le monde, à quelques nuances près, est d'accord sur la manière de prendre en compte les exigences de sécurité pour un sol purement frottant, le débat est largement ouvert quant à la manière de prendre en compte la contribution de la cohésion.

La pratique assez courante de non prise en compte la cohésion effective est contestable, et il n'y a pas forcément lieu de lui appliquer un coefficient partiel différent de celui appliqué à $\tan (\varphi)$ (même si, au paragraphe suivant, nous reprenons les errements usuels). Par contre, dans l'immense majorité des cas, il faut considérer que le sol est dénué de résistance en traction (le domaine des tractions n'est généralement pas l'objet d'essais, et le comportement y est rarement ductile).

Pour illustrer les effets de la coupure de résistance en traction, on peut se reporter à la figure 22 qui 
montre, dans un cas particulier, la relation entre la résistance et la longueur d'un ancrage isolé disposant d'un frottement latéral surabondant, avec trois approches : en négligeant la cohésion (a), avec coupure de la résistance en traction (b), avec un critère complet (c).

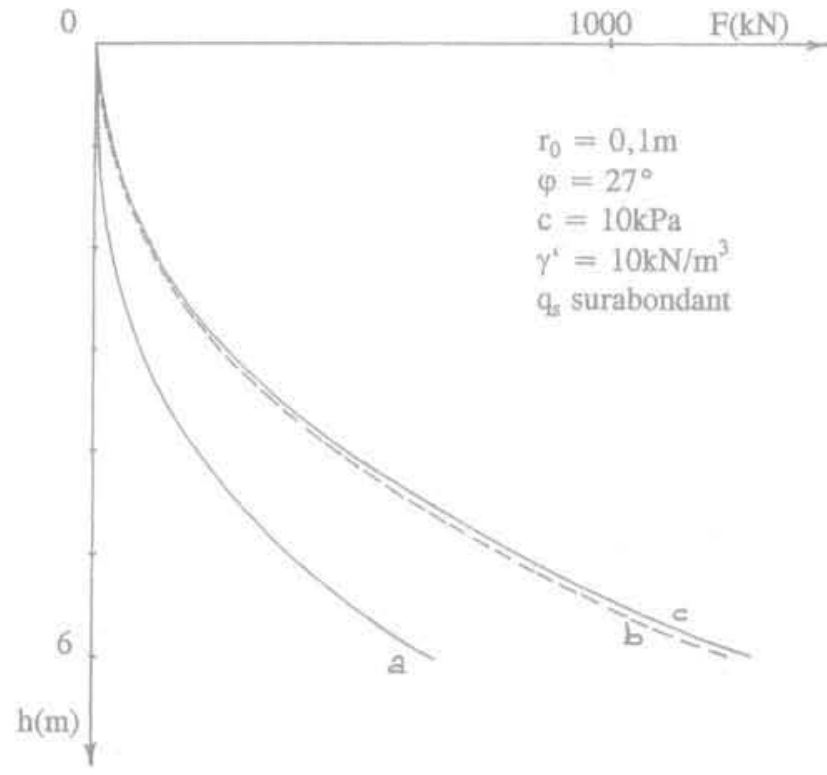

FiG. 22 Incidence de la cohésion, avec ou sans coupure de résistance en traction.

Influence of cohesion, with or without the tension truncated criterion.

On constate que, au moins dans le cas présenté (et les quelques autres cas que nous avons testés), la coupure de résistance en traction ne réduit que très peu la contribution de la cohésion à la résistance géotechnique d'un ancrage vertical. Dans le cas évoqué au début de l'article, la coupure de résistance en traction réduit la hauteur limite d'une falaise verticale dans un rapport de 3,85 environ à 2. Il apparaît donc que la résistance en traction joue un rôle plus ou moins important selon la nature de l'ouvrage considéré. On a donc deux pistes pour faire avancer le débat relatif à la valeur du coefficient partiel relatif à la cohésion :

- soit on pratique la coupure de résistance en traction et le coefficient partiel peut être le même sur c et sur $\tan (\varphi)$; ceci suppose que l'on sache prendre en compte dans les calculs les effets de la coupure de résistance en traction :

- soit on effectue des calculs utilisant le critère complet, et on multiplie le coefficient partiel sur $\tan (\varphi)$ par un coefficient complémentaire couvrant les effets de la coupure de résistance en traction pour définir le coefficient partiel relatif à c, le coefficient complémentaire étant différent pour chaque nature d'ouvrage, et résultant d'une étude paramétrique ; retenons qu'il n'est pas raisonnable d'appliquer, dans le cas du critère complet, un coefficient partiel sur la cohésion indépendant de la nature de l'ouvrage.

\section{0}

\section{Justifications de résistance}

La justification des ouvrages par le calcul passe aujourd'hui par l'application de la méthode des états limites.
La résistance géotechnique tient compte de la souspression, du poids volumique du sol déjaugé, de sa cohésion et de son angle de frottement interne.

Pour justifier la longueur des ancrages, on doit vérifier l'équilibre statique en conditions EE. Les coefficients partiels multiplicateurs réglementaires sont, pour les ouvrages courants:

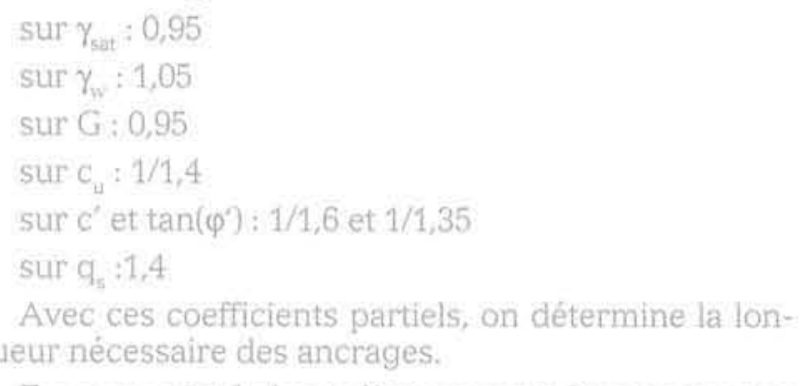

En tout point le long d'un ancrage (et notamment en sa liaison avec le radier), l'armature (en acier) doit être en mesure de résister à la traction associée à ces coefficients partiels sans que sa contrainte n'excède $f_{f} /(1,2.1,15)$; si l'armature n'est équipée d'aucune protection spécifique contre la corrosion, il faut évidemment à ce stade tenir compte d'une perte de matière fonction de l'environnement et de la durée de service.

\section{1}

\section{Conclusion}

Dans tous les cas de sol homogène dont la résistance au cisaillement est correctement identifiée, la méthode cinématique du calcul à la rupture permet de traiter «proprement » l'étude de la résistance géotechnique des ancrages verticaux, qu'ils soient isolés ou inclus dans une trame (avec dans ce cas les imprécisions modestes associées à la modélisation axisymétrique)

Elle permet de prendre en compte sans difficulté majeure un critère de Coulomb tronqué en traction ; il est très facile d'adapter le programme proposé à d'autres critères de résistance du sol, tels qu'un critère parabolique $\left(\tau_{\text {m }}=\right.$ A. $\left.\sigma^{\downarrow}\right)$, ou bien à des sols anisotropes (sous réserve que l'anisotropie respecte l'axisymétrie, bien entendu).

Le schéma de calcul peut aisément être adapté à des ancrages qui ne sont pas scellés sur toute leur longueur.

L'approche est évidemment plus laborieuse dans divers cas de sols stratifiés (les strates étant horizontales), et il reste à résoudre le cas de strates inclinées...

Nous rappellerons, pour finir, que :

- la méthode cinématique du calcul à la rupture est une approche rígoureuse, mais « par l'extérieur », et ne fournit donc des conclusions fiables qu'entre les mains de projeteurs expérimentés, explorant soigneusement. des familles pertinentes de mécanismes de rupture; - le comportement des matériaux et des interfaces est supposé plastique ou élastoplastique sans radoucissement; lorsqu'on est en présence de comportements radoucissants, la prise en compte de caractéristiques de pic peut conduire à une large surévaluation de la résistance géotechnique, tandis que la prise en compte de caractéristiques résiduelles conduit a priori à une sous-évaluation; il est le plus souvent raisonnable de 
baser les justifications sur les caractéristiques résiduelles (sauf si une modélisation plus sophistiquée permet de prendre en compte des lois de comportement plus complètes et représentatives que les seuls paramètres de résistance...);

- le frottement latéral limite est en principe déterminé au moyen d'essais d'arrachement (essai par paliers de force, ou à vitesse d'arrachement constante) ; nous insisterons sur l'intérêt de disposer d'informations sur le comportement post-pic (donc sur l'intérêt des essais à vitesse d'arrachement constanté, ou sur la nécessité de compléter les essais par paliers de chargement par une procédure à vitesse constante après constat de défaillance);

- dans les cas d'ouvrages pour lesquels la part de variation cyclique de la traction est notable par comparaison avec la traction quasi permanente, le frottement latéral limite est susceptible d'évoluer à la baisse (fatigue) ; on manque cruellement d'indications réglementaires ou expérimentales, mais il est certain que l'on doit alors prendre en compte un coefficient partiel relatif à $\mathrm{q}_{8}$ plus grand que 1,4 lorsque $\mathrm{q}_{8}$ est déterminé par un essai monotone, car 1,4 ne couvre pas la fatigue; des recherches sont indispensables à ce propos.

\section{Bibliographie}

CCTG, fascicule $62 \mathrm{~V}$, Règles techriques de conception et de calcul des fondations des ouvrages de génie civil.

DTU 13.2. Fondations profondes pour le bâtiment.

ENV 1997 (Eurocode 7), « Calcul géotechnique $x$.

Recommandations TA 95, a Tirants d'ancrage $x$.

Buhan (de) P.. Salençon J. - a A comprehensive stability analysis of soil nailed structures D. European Journal of Mechanics, a/solids, vol. 12, n 3 3, 1993. Cher W.F. - Limit analysis and soil plasticity. Developments in geotechnical engineering, vol. 7 . Elsevier scientific publishing company, 1975 .

Pecker A., Salençon J. - « Ultimate bearing capacity of shallow foundations under inclined and eccentric loads. Part 1. Part II. 刃. European Journal of Mechanics, a'solids, vol. 14, n³ $3,1995$.
Salencon J. - Calcul à la rupturè et analyse limite. Presses des Ponts et Chaussées. 1983.

Salençon J. - « Calcul à la rupture appliqué au calcul des ouvrages x. Cours de l'ENPC

Salencon J. - « Approche théorique du calcul aux états limites ultimes n. RMA 28.

Salençon J. - "An introduction to the Yield clesing theory and its applications to soil mechanics x - European Journal of Mechanics, a/solids, vol. 9, n 5, 1991.

\section{Annexe 1}

\section{Ancrage isolé scellé dans un sol bicouche sans cohésion}

Nous avons vu dans le texte que, dans un sol homogène, la courbe pertinente $\mathrm{F}_{\text {lim3 } 3}$ est confondue avec la courbe $F_{\text {mint }}$ (poids du bloc) jusqu'à ce que la tangente à $F_{\text {limi1 }}$ soit parallèle à $F_{\text {lim2 }}$ (profondeur que nous avons qualifiée de critique), et elle suit alors une droite parallèle à $F_{\text {lim? }}$.

Dans l'exemple présenté ci-dessous, un sol peu performant recouvre un sol plus résistant :

- sol de couverture, épaisseur $4 \mathrm{~m}, \gamma=8 \mathrm{kN} / \mathrm{m}^{3}, \varphi=20^{\circ}$, $\mathrm{q}_{\mathrm{s}}=80 \mathrm{kPa}$;

- couche sous-jacente, $\gamma=10 \mathrm{kN} / \mathrm{m}^{3}, \varphi=35^{\circ}$, $\mathrm{q}_{\mathrm{s}}=400 \mathrm{kPa}$.

L'ancrage est de diamètre $0,15 \mathrm{~m}$.
On envisage des valeurs croissantes de h, la longueur de l'ancrage.

La longueur critique pour les caractéristiques du sol de couverture est égale à $3,365 \mathrm{~m}$. Pour un ancrage de longueur inférieure à $3,365 \mathrm{~m}$, le schéma de rupture est un cône de hauteur $\mathrm{h}$; pour un ancrage plus long, c'est un cône de hauteur $3,365 \mathrm{~m}$, et au-delà la rupture suit l'interface sol/ancrage.

Lorsque l'ancrage atteint la couche sous-jacente, le frottement latéral limite est beaucoup plus important, et dès la longueur de $4,058 \mathrm{~m}$, c'est à nouveau un schéma de rupture conditionné par le sol (troncs de cônes superposés) jusqu'à une nouvelle profondeur critique égale à $4,778 \mathrm{~m}$, au-delà de laquelle la rupture passe par l'interface sol/ancrage.

Le poids du sol intervient donc seul pour le domaine de longueur d'ancrage $[0 ; 3,365]$ U $[4,048$; $4,778]$; en dehors de ce domaine, la rupture passe aussi par l'interface sol/ancrage.

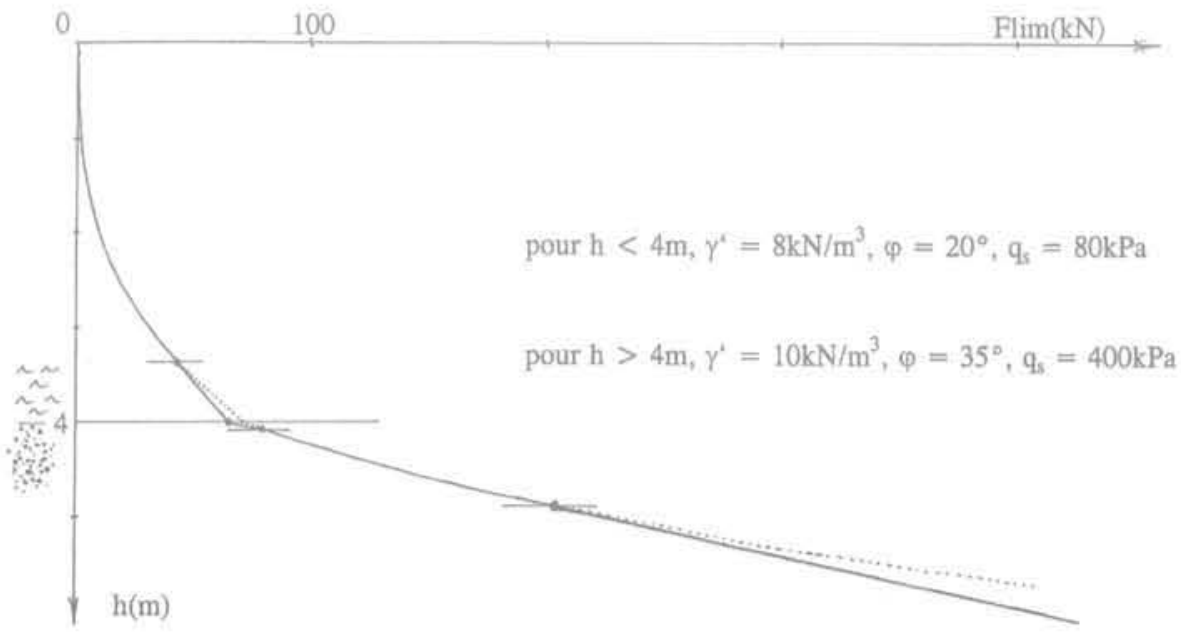

FG. A1 Notion de longueur critique en sol stratifié.

Notion of critical length in a multilayer soil. 


\section{Annexe 2}

\section{Calcul du volume de sol solidaire d'un ancrage au sein d'une trame pour un sol sans cohésion}

On cherche une formulation analytique de la différence de volume entre le cylindre de hauteur égale à la longueur de l'ancrage et le bloc limité par un cône de même axe.

La ligne d'intersection entre le cône et l'une des faces latérales du cylindre à base carrée est un arc d'hyperbole; dans le plan XAz défini sur la figure A2, l'équation de la ligne est :

$$
z=\left(a^{3} / 4+x^{2}\right)^{0,5 / \tan (\varphi)}
$$

La surface hachurée A est obtenue par intégration de $z$ pour $x$ variant entre 0 et a/2.

La primitive a pour expression :

$\left[a^{2} \cdot \ln \left(x+\left(x^{2}+a^{2} / 4\right)^{0.5}\right)+4 \cdot x \cdot\left(x^{2}+a^{2} / 4\right)^{0.5}\right] / 8 / \tan (\varphi)$

On en tire :

$$
A=a^{2} \cdot\left(2^{0.5}+\ln \left(1+2^{0,5}\right)\right) / 8 / \tan (\varphi)
$$

Soit le volume cherché :

$$
\mathrm{V}_{0}=8 \cdot \mathrm{A} \cdot \mathrm{a} / 6
$$
rayon :

La cellule axisymétrique de même section a pour conduisant à :

$$
\mathrm{r}=\mathrm{a} / \pi^{0,5}
$$

$$
\begin{gathered}
V_{1}=2 \cdot \pi / 3 / \tan (\varphi) \cdot r^{3} \\
V_{1}=a 3 / \tan (\varphi) \cdot 2 / 3 / \pi^{0.5}
\end{gathered}
$$

On obtient ainsi $\mathrm{V}_{0}=1,0172 . \mathrm{V}_{1}$

Un ensemble d'ancrages répartis selon une trame carrée, avec une trame et une longueur d'ancrage telles que les intersections des cònes aient lieu dans le sol, associe au radier une plaque de sol dont l'épaisseur moyenne est :

$$
e_{0}=L-0,382598 \cdot a / \tan (\varphi)
$$

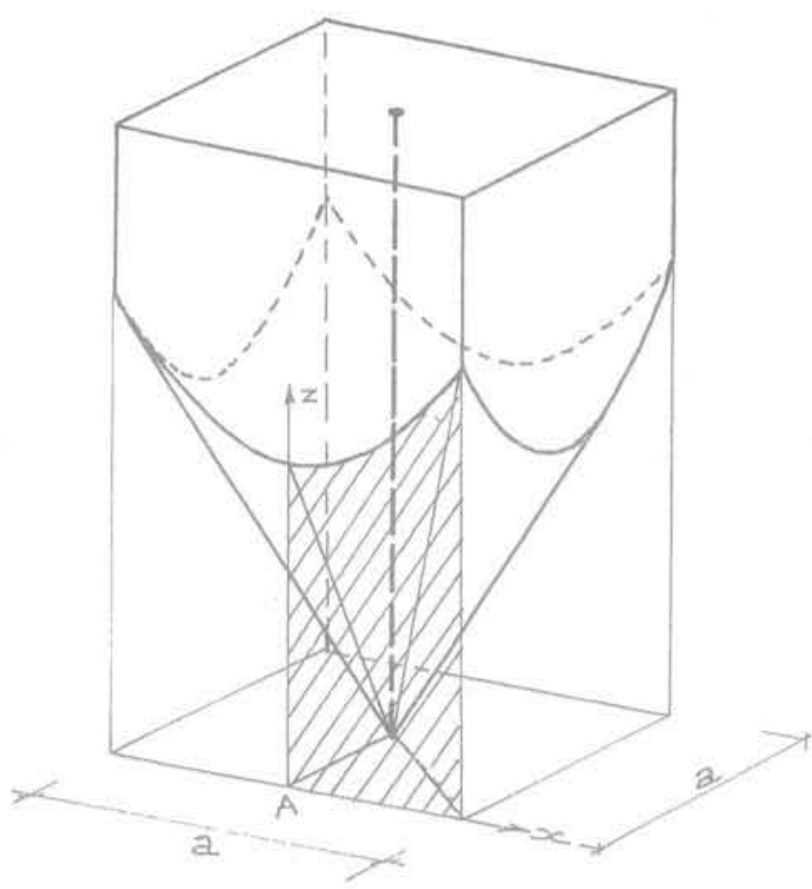

FIG.A2 Repérage pour le calcul du volume. Sketch for the volume calculation.

En assimilant chaque cellule à une cellule circulaire de même section, on a :

$$
e_{1}=L-0,376126 . \mathrm{a} / \tan (\varphi)
$$

Pour $\mathrm{a}=3 \mathrm{~m}$ et $\varphi=30^{\circ}, \mathrm{e}_{1}=1,988 \mathrm{~m}$ et $\mathrm{e}_{2}=1,954 \mathrm{~m}$, soit $34 \mathrm{~mm}$ de différence.

Dans le cas d'une trame triangulaire, on obtient à l'issue des calculs :

$$
\begin{aligned}
& e_{0}=L-0,303993 \cdot a / \tan (\varphi) \\
& e_{1}=L-0,303131 \cdot a / \tan (\varphi)
\end{aligned}
$$

Pour $\mathrm{a}=3 \mathrm{~m}$ et $\varphi=30^{\circ}$, $\mathrm{e} 1=1,580 \mathrm{~m}$ et $\mathrm{e} 2=1,575 \mathrm{~m}$, soit $5 \mathrm{~mm}$ de différence.

Le programme ANCRCOUR ne présente pas d'utilité pratique ; il est listé dans la mesure où il comporte un algorithme d'optimisation de la ligne directrice d'une surface de rupture à laquelle on impose la position des extrémités, algorithme qui peut être utile pour des cas de sols stratifiés par exemple (ce programme a été utilisé pour constater l'équivalence avec une approche plus rapide dans le cas de surfaces de rupture émergeant en surface du massif).
Le programme ANCRRAD permet de déterminer la résistance géotechnique d'un ancrage au sein d'une trame, dans le cas d'un sol homogène et isotrope. 
REM $\star \star \star *$ ANCRRAD $\star * *$

REM $* * *$ capacite d'un ancrage vertical dans trame sous radier

$\mathrm{pi}=3.141593$

10 INPUT "inclusion, rayon $(\mathrm{m})="$; ro INPUT " frott. lat. lim. $(\mathrm{kPa})=" ;$ qs INPUT " Iongueur $(\mathrm{m})={ }^{n} ; 1$

INPUT "modele axisym., rayon $(\mathrm{m})="$; $r 2$

INPUT "massif de sol, poids vol. $(\mathrm{kN} / \mathrm{m} 3)=" ; \mathrm{g}$

INPUT " cohesion ( $\mathrm{kPa})=" ; \mathrm{c}$

INPUT " angle de frott. interne $(\not / 2)=" ;$ fid: $f i=f i d *$ pi $/ 180$

$\mathrm{tf}=\operatorname{TAN}(\mathrm{pi} / 4-\mathrm{fi} / 2)$

rcrit $=\operatorname{SQR}(2 * r 0 * \mathrm{qs} / \mathrm{g}+\mathrm{rO} * 2)$

IF $r 2>$ rcrit GOTO 200

$r 1=-c / g *(1+\operatorname{SIN}(f i))+\operatorname{SQR}((c / g *(1+\operatorname{SIN}(f i))) \wedge 2+r 2 \wedge 2)$

IF $r 0>r 1$ THEN $r a=r 0: y=0: f=0:$ GOTO 100

$y=(r 1-r 0) / \operatorname{TAN}(f i): r a=r 1$

$\mathrm{f}=\mathrm{pi} *(\mathrm{r} 1 \wedge 2-r 0 \wedge 2) * \mathrm{c} / \mathrm{TAN}(\mathrm{fi})$

$f=f-p i * g / 3 *(3 * r 2 \wedge 2-r 1 \wedge 2-r 0 * r 1-r 0 \wedge 2) * y$

$100 k=r 2 / \mathrm{ra}: r g=r a: u=2 * c / g / \operatorname{TAN}(p i / 4-\mathrm{fi} / 2)$

FOR $i=1$ TO 50

$r d=r a * k \wedge(.02 * i)$

$r m=.5 *(r d+r g): d r=r d-r g$

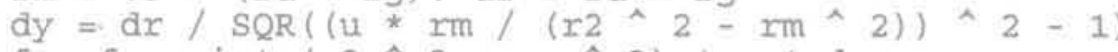

$\mathrm{f}=\mathrm{f}-\mathrm{pi} *(\mathrm{r} 2 \times 2-\mathrm{rm} \wedge 2) * \mathrm{~g} * \mathrm{dy}$

$f=f+2 * p i * r m * c *(\operatorname{SQR}(d r * 2+d y \wedge 2)-d r) / t f$

$y=y+d y: r g=r d$

NEXT

IF $y>1$ THEN PRINT "ancrage court": GOTO 400

$t=p i * r 2 \wedge 2 * 1 * g+\hat{f}$

PRINT "traction limite $=" ; t ;{ }^{\prime} \mathrm{kN} "$

PRINT $r 2, t, y, f, r c r i t, r a, r 2$

GOTO 10

200 r2 $=$ rerit

$r 1=-c / g *(1+\operatorname{SIN}(f i))+\operatorname{SQR}((c / g *(1+\operatorname{SIN}(f i))) \wedge 2+r 2 \wedge 2)$

IF $r O>r 1$ THEN $r a=r 0: y=0: f=0:$ GOTO 300

$y=(r 1-r 0) / \operatorname{TAN}(f i): r a=r 1$

$f=p i *(r i \wedge 2-r 0 \wedge 2) * c / T A N(f i)$

$f=f-p i * g / 3 *(3 * r 2 * 2-r 1 \wedge 2-r 0 * r I-r 0 \wedge 2) * y$

$300 \mathrm{k}=\mathrm{r} 2 / \mathrm{ra}: \mathrm{rg}=\mathrm{ra}: \mathrm{u}=2 * \mathrm{c} / \mathrm{g} / \mathrm{TAN}(\mathrm{pi} / 4-\mathrm{fi} / 2)$

FOR $i=1$ TO 50

$r d=r a * k^{\wedge}(.02 * i)$

$r m=.5 *(r d+r g): d r=r d-r g$

$d y=d r / \operatorname{SQR}((u * r m /(r 2 \wedge 2-r m \wedge 2)) \wedge 2-1)$

$f=f-p i *(r 2 \wedge 2-r m \wedge 2) * g * d y$

$f=f+2 * p i * r m * c *(S Q R(d r * 2+d y \wedge 2)-d r) / t f$

$y=y+d y: r g=r d$

NEXT

IF $\mathrm{y}>1$ THEN PRINT "ancrage court": GOTO 400

$t=p i * r 2 * 2 * y * g+f+p i * r 0 *(2 * q s+r 0 * g) *(1-y)$

PRINT "2 * 1'ancrage fonctionne comme un ancrage isole"

PRINT " traction limite $=" ; t$; "kN"

PRINT $r 2, t, y, f, r c r i t, r a, r 2$

GOTO 10

$400 \mathrm{dr2}=.5 *(r 2-r 0): r 2=r 2-\mathrm{d} 2 \mathrm{r2}$

FOR $j=1$ TO 20

$r 1=-\mathrm{c} / \mathrm{g} *(1+\operatorname{SIN}(f i))+\operatorname{SQR}((\mathrm{c} / \mathrm{g} *(1+\operatorname{SIN}(\mathrm{fi}))) \wedge 2+r 2 \wedge 2)$

IF $r 0>r 1$ THEN $r a=r 0: y=0: f=0:$ GOTO 500

$y=(r 1-r 0) / \operatorname{TAN}(f i): r a=r 1$ 

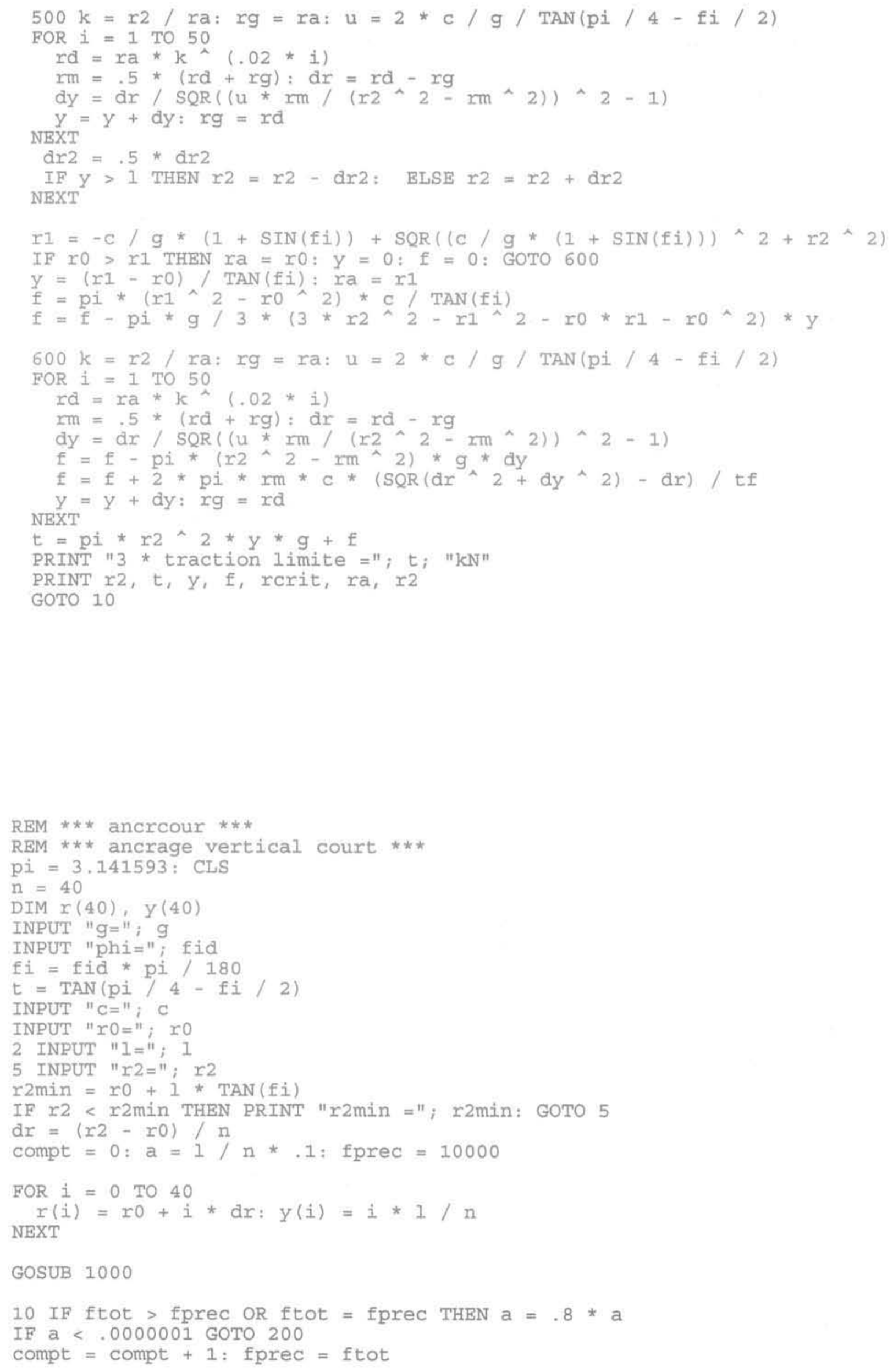


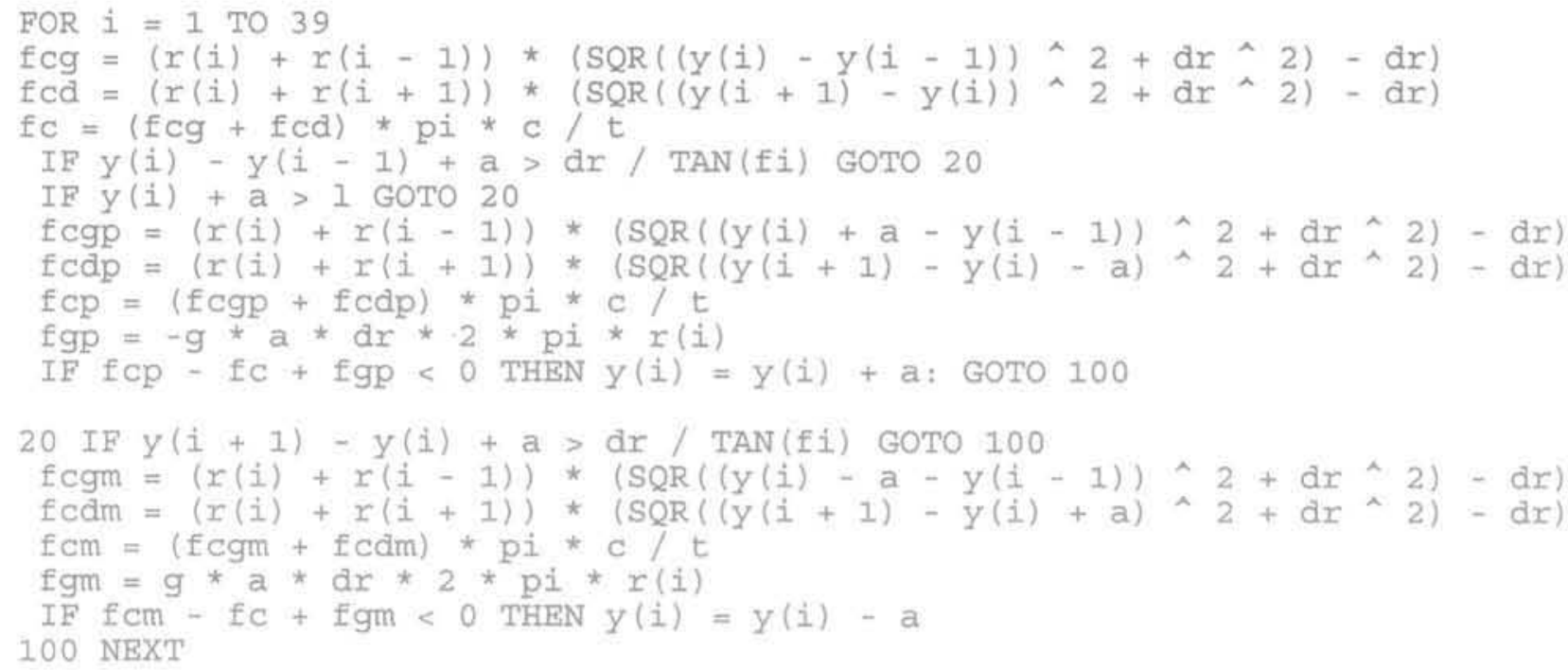

GOSUB 1000

GOTO 10

200 FOR $i=1$ TO 20: PRINT USING "\#\#\#.\#\#\#"; $r(2$ * i);

PRINT USING "\#\#\#.\#\#\#"; y(2*i); : PRINT" ", : NEXT: PRINT GOTO 2

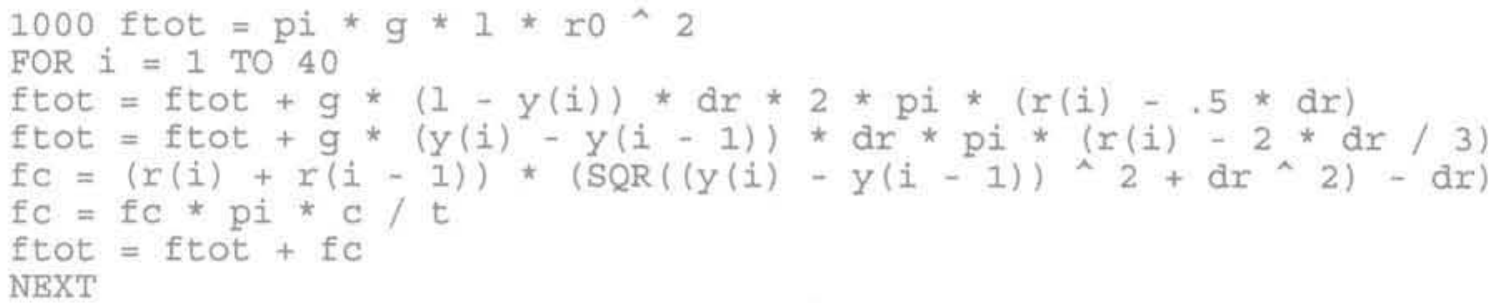

LOCATE 9, 40: PRINT compt, a

LOCATE 10, 40: PRINT USING "\#\#\#\#.\#\#\#\#\#"; ftot

RETURN 